\title{
Constructing smooth and fully faithful tropicalizations for Mumford curves
}

\author{
Philipp Jell ${ }^{1}$ \\ Published online: 10 August 2020
}

(c) The Author(s) 2020

\begin{abstract}
The tropicalization of an algebraic variety $X$ is a combinatorial shadow of $X$, which is sensitive to a closed embedding of $X$ into a toric variety. Given a good embedding, the tropicalization can provide a lot of information about $X$. We construct two types of these good embeddings for Mumford curves: fully faithful tropicalizations, which are embeddings such that the tropicalization admits a continuous section to the associated Berkovich space $X^{\text {an }}$ of $X$, and smooth tropicalizations. We also show that a smooth curve that admits a smooth tropicalization is necessarily a Mumford curve. Our key tool is a variant of a lifting theorem for rational functions on metric graphs.
\end{abstract}

Keywords Tropical geometry $\cdot$ Smooth tropical curves $\cdot$ Mumford curves $\cdot$ Extended skeleta $\cdot$ Faithful tropicalization

Mathematics Subject Classification Primary: 14T05; Secondary: 14G22 · 32P05

\section{Introduction}

Let $K$ be a field that is algebraically closed and complete with respect to a nonarchimedean non-trivial absolute value. Given a closed subvariety $X$ of a toric variety $Y$ over $K$, one can associate a so-called tropical variety $\operatorname{Trop}(X)$ which is a polyhedral complex. Note however, that $\operatorname{Trop}(X)$ is not an invariant of $X$, but depends on the embedding into $Y$.

In good situations, $\operatorname{Trop}(X)$ can retain a lot of information about $X$. Let us mention here work by Katz, Markwig and Markwig on the $j$-invariant of elliptic curves [21,

The author was supported by the DFG Research Fellowship JE 856/1-1 and by the Institute Mittag-Leffler and the "Vergstiftelsen".

\footnotetext{
$\varangle \quad$ Philipp Jell

philipp.jell@ur.de

1 Universität Regensburg, 93040 Regensburg, Germany
} 
22] and work by Itenberg, Mikhalkin, Katzarkov and Zharkov on recovering Hodge numbers in degenerations of complex projective varieties [17].

In the latter work, a smoothness condition for tropical varieties in arbitrary codimension appears: a tropical variety is called smooth if it is locally isomorphic to the Bergman fan of a matroid. (See Definition 2.6 for an equivalent definition for curves.) For tropical hypersurfaces, this is equivalent to the associated subdivision of the Newton polytope being a primitive triangulation, which is the definition of smoothness that is generally used for tropical hypersurfaces [17, Remark p. 24].

The definition in [17] is motivated by complex analytic geometry. A complex variety is smooth if it is locally isomorphic to open subsets of $\mathbb{C}^{n}$ in the analytic topology. Bergman fans of matroids are the local models for linear spaces in tropical geometry, thus it makes sense to call a tropical variety smooth if it is locally isomorphic to the Bergman fan of a matroid.

This smoothness condition has been shown to imply many tropical analogues of classical theorems from complex and algebraic geometry, for example intersection theory, Poincaré duality and a Lefschetz $(1,1)$-theorem [18,19,31].

In this paper, we investigate the question for which smooth projective curves there exist closed embeddings $\varphi$ into toric varieties such that $\operatorname{Trop}_{\varphi}(X):=\operatorname{Trop}(\varphi(X))$ is smooth. The answer turns out to be Mumford curves (see Definition 2.11). Indeed, we show that for these curves we can "repair" any given embedding by passing to a refinement (see Definition 2.16 for a definition of refinement).

Theorem A (Theorem 4.10, Theorem 5.6) Let X be a smooth projective curve of positive genus. Then the following are equivalent:

(i) $X$ is a Mumford curve.

(ii) There exists a closed embedding $\varphi: X \rightarrow Y$ for a toric variety $Y$ that meets the dense torus such that $\operatorname{Trop}(\varphi(X))$ is a smooth tropical curve.

(iii) Given a closed embedding $\varphi: X \rightarrow Y$ of $X$ into a toric variety $Y$ that meets the dense torus, there exists a refinement $\varphi^{\prime}: X \rightarrow Y^{\prime}$ of $\varphi$ such that $\operatorname{Trop}\left(\varphi^{\prime}(X)\right)$ is a smooth tropical curve.

Denote by $X^{\text {an }}$ the Berkovich analytification of $X$ [3]. We give alternative characterizations of Mumford curves in terms of $X^{\text {an }}$ in Remark 2.12. Theorem A, specifically the equivalence of (i) and (ii), may be viewed as an alternative characterization that is purely tropical.

Payne showed in [30, Theorem 4.2] that we have a homeomorphism

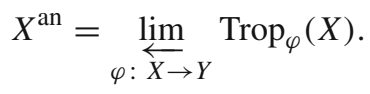

Theorem A shows that if $X$ is a Mumford curve we can let the limit on the right hand side as well run only over closed embeddings $\varphi$ such that $\operatorname{Trop}_{\varphi}(X)$ is a smooth tropical curve, meaning the smoothness on the left hand side is reflected on the right hand side.

Another often used property of tropicalizations is faithfulness. For curves this means that given a finite skeleton $\Gamma$ of $X^{\text {an }}$, one requires that $\varphi_{\text {trop }}:=\operatorname{trop} \circ \varphi^{\text {an }}$ is a homeomorphism from $\Gamma$ onto its image, preserving the piecewise linear structure. Existence 
of faithful tropicalizations was proved by Baker, Payne and Rabinoff for curves and generalized to higher dimension by Gubler, Rabinoff and Werner [6,13]. For further work on faithful tropicalizations see for example [10,24,25,33].

Baker, Payne and Rabinoff also introduced so-called completed extended skeleta for curves. For a smooth projective curve $X$, these are metric subgraphs $\Sigma$ of $X^{\text {an }}$, potentially with edges of infinite length, that come with a canonical retraction $\tau: X^{\text {an }} \rightarrow \Sigma$. Given a closed embedding $\varphi: X \rightarrow Y$ for $Y$ a toric variety with dense torus $T$, there exists an associated complete skeleton $\Sigma(\varphi)$, which has the property that $\varphi_{\text {trop }}$ factors through the retraction $\tau: X^{\text {an }} \rightarrow \Sigma(\varphi)$ (see Definition 2.17). Denote by $X^{\circ}:=\varphi^{-1}(T)$. We call $\varphi_{\text {trop }}$ fully faithful if $\varphi_{\text {trop }}$ maps $\Sigma(\varphi)$ homeomorphically onto its image and is an isometry when restricted to $\Sigma(\varphi) \cap X^{\circ \text {, an }}$. Note that this is much stronger than a faithful tropicalization, since by definition the image of $\Sigma(\varphi)$ is $\operatorname{Trop}_{\varphi}(X)$.

We prove the following fully faithful tropicalization result.

Theorem B (Theorem 4.6) Let $X$ be a Mumford curve and $\varphi: X \rightarrow Y$ a closed embedding into a toric variety $Y$ that meets the dense torus. Then there exists a refinement $\varphi^{\prime}$ of $\varphi$ that is fully faithful.

As a direct consequence of the fact that $\varphi^{\prime}$ is fully faithful, we obtain a continuous section $s: \operatorname{Trop}_{\varphi^{\prime}}(X) \rightarrow X^{\text {an }}$ of $\varphi_{\text {trop }}^{\prime}$ by composing the inverse of $\left.\varphi_{\text {trop }}^{\prime}\right|_{\Sigma\left(\varphi^{\prime}\right)}$ with the inclusion of $\Sigma\left(\varphi^{\prime}\right)$ into $X^{\text {an }}$ (see Corollary 4.7). Such sections, though only defined on subsets of $\operatorname{Trop}_{\varphi}(X)$, were also constructed in [6, Theorem 5.24] and [14, Theorem 8.15].

For reader interested in effective bounds on the dimensions of the ambient toric varieties, let us mention [12], where Gunn and the author construct fully faithful tropicalizations in ambient dimension 3, and also give bounds on the ambient dimensions for smooth tropicalizations.

We prove Theorem B as a first step to prove Theorem A, more precisely that (i) implies (iii) therein. Our techniques to prove these results are based on the following lifting theorem for rational functions on metric graphs, which is a variant of a theorem by Baker and Rabinoff [7, Theorem 1.1]. The relevant notions are recalled in Sect. 2.5.

Theorem C (Theorem 3.2) Let X be a Mumford curve and $\Gamma$ be a finite skeleton with retraction $\tau$. Let $D \in \operatorname{Div}(X)$ be a divisor of degree $g$ and let $B=p_{1}+\cdots+p_{g} \in$ $\operatorname{Div}(\Gamma)$ be a break divisor such that $\tau_{*} D-B$ is a principal divisor on $\Gamma$. Assume that $B$ is supported on two-valent points of $\Gamma$. Then there exist $x_{i} \in X(K)$ such that $\tau_{*} x_{i}=p_{i}$ and such that $D-\sum_{i=1}^{g} x_{i}$ is a principal divisor on $X$.

Theorem $\mathrm{C}$ is of independent interest, since, given a skeleton of $X$, it enables one to construct closed embeddings with nice tropicalizations. We treat an example of this in Example 3.5 for a genus 1 Mumford curve (also called a Tate curve).

We give an idea of the proof of Theorem B, which is carried out in Sect. 4.2. Given an edge $e$ of $\Sigma(\varphi)$, using Theorem C, we construct a rational function $f_{e} \in$ $K(X)^{*}$ in such a way that $\log |f|$ has slope 1 along $e$. Considering the embedding $\varphi^{\prime}:=\left(\varphi, f_{e}\right): X \rightarrow Y \times \mathbb{P}^{1}$, this ensures that $\varphi_{\text {trop }}^{\prime}$ maps $e$ homeomorphically onto its image and that the corresponding stretching factor equals 1 (see Definition 2.18 for the 
definition of stretching factor). Using a good choice of $D \in \operatorname{Div}(X)$ and $B \in \operatorname{Div}(\Gamma)$, Theorem $\mathrm{C}$ moreover allows us to construct $f_{e}$ in such a way that the same holds for all edges of $\Sigma\left(\varphi^{\prime}\right)$ that are not contained in $\Sigma(\varphi)$. Doing so for every edge of $\Sigma(\varphi)$, we obtain Theorem B.

In Sect. 4.3, we proceed similarly for smoothness and thus prove that (i) implies (iii) in Theorem A.

In Sect. 5 we prove that, for a smooth projective curve $X$, the existence of a closed embedding with a smooth tropicalization already implies that $X$ is a Mumford curve. The key result we use is a joint observation by Mikhalkin, Sturmfels and Ziegler [26], which states that a variety whose tropicalization is a tropical linear space is actually a linear space (see Theorem 5.4). The version of the theorem we use was proved by Katz and Payne [23] and works for trivially valued fields in any characteristic (see Theorem 5.4). We also show that if $\operatorname{Trop}_{\varphi}(X)$ is smooth then $\varphi_{\text {trop }}$ is necessarily fully faithful (see Theorem 5.7).

\section{Conventions}

Throughout, $K$ will be an algebraically closed field that is complete with respect to a non-archimedean non-trivial absolute value $|\cdot|_{K}$. We denote the value group by $\Lambda:=\log \left|K^{\times}\right|_{K}$, the valuation ring by $K^{\circ}$ and the residue field by $\tilde{K}$. A variety over $K$ is a separated reduced irreducible scheme of finite type and a curve is a onedimensional variety. $X$ will be a smooth projective curve over $K$. We will denote finite skeleta of $X$ by $\Gamma$ and completed extended skeleta in the sense of [5] by $\Sigma$. We will generally denote toric varieties by $Y$ and their dense tori by $T$.

\section{Preliminaries}

\subsection{Tropical toric varieties and tropical curves}

Let $N$ be a free abelian group of rank $n, M:=\operatorname{Hom}_{\mathbb{Z}}(N, \mathbb{Z})$ its dual, $N_{\mathbb{R}}:=N \otimes \mathbb{R}$ and $\Delta$ a rational pointed fan in $N_{\mathbb{R}}$. We write $\mathbb{T}:=\mathbb{R} \cup\{-\infty\}$.

For $\sigma \in \Delta$ we define the monoid $S_{\sigma}:=\{\varphi \in M \mid \varphi(v) \geq 0$ for all $v \in \sigma\}$ and write $N(\sigma):=N_{\mathbb{R}} /\langle\sigma\rangle_{\mathbb{R}}$, where $\langle\sigma\rangle_{\mathbb{R}}$ denotes the real vector space spanned by $\sigma$. We write

$$
N_{\Delta}=\coprod_{\sigma \in \Delta} N(\sigma) .
$$

We endow $N_{\Delta}$ with a topology in the following way:

For $\sigma \in \Delta$ write $N_{\sigma}=\bigsqcup_{\tau \prec \sigma} N(\tau)$. This is naturally identified with $\operatorname{Hom}_{\text {Monoids }}\left(S_{\sigma}, \mathbb{T}\right)$. We give $N_{\sigma}$ the subspace topology of $\mathbb{T}^{S_{\sigma}}$. For $\tau \prec \sigma$, the space $\operatorname{Hom}\left(S_{\tau}, \mathbb{T}\right)$ is naturally identified with the open subspace of $\operatorname{Hom}_{\text {Monoids }}\left(S_{\sigma}, \mathbb{T}\right)$ of maps that map $\tau^{\perp} \cap M$ to $\mathbb{R}$. We define the topology of $N_{\Delta}$ to be the one obtained by gluing along these identifications. 
Definition 2.1 We call the space $N_{\Delta}$ a tropical toric variety.

The space $N_{\Delta}$ is sometimes called the canonical compactification of $N_{\mathbb{R}}$ with respect to $\Delta$. Note that $N_{\Delta}$ contains $N_{\mathbb{R}}$ as a dense open subset.

Example 2.2 Let $N=\mathbb{Z}^{n}$ with basis $x_{1}, \ldots, x_{n}$ and $\Delta$ be the complete fan whose rays are spanned by $-x_{1}, \ldots,-x_{n}$ and $x_{0}:=\sum x_{i}$. For any $d$-rays there is a face $\sigma$ of dimension $d$ that contains exactly these rays. Then $N(\sigma)$ is an $n-d$-dimensional vector space. The topology is such that $N_{\Delta}$ is homeomorphic to an $n$-simplex, where $N(\sigma)$ is identified with the relative interior of a $n-d$-dimensional simplex in the boundary. For example, $N_{\mathbb{R}}$ corresponds to the vertex at the origin in $\Delta$ and forms the interior of $N_{\Delta}$ when we view $N_{\Delta}$ as a simplex.

However, we will heavily use the structure of $N_{\mathbb{R}}$ as a vector space, so we generally view $N_{\Delta}$ as a compactification of $N_{\mathbb{R}}$ by strata that are infinitely far away.

Definition 2.3 Let $\mathcal{C}$ be a one dimensional $\Lambda$-rational polyhedral complex in $N_{\mathbb{R}}$. For an edge $e$ (i.e. a one-dimensional polyhedron) of $\mathcal{C}$ we denote by $\mathbb{L}(e)=\left\{\lambda\left(u_{1}-u_{2}\right) \mid\right.$ $\left.u_{1}, u_{2} \in e, \lambda \in \mathbb{R}\right\}$ the linear space of $e$. Since $X$ is $\Lambda$-rational, $\mathbb{L}(e)$ contains a canonical lattice which we denote by $\mathbb{Z}(e)$.

For a vertex $v$ of $e$ we denote by $w_{v, e}$ the unique generator of $\mathbb{Z}(e)$ that points in $e$ away from $v$.

We call $\mathcal{C}$ weighted if every edge is equipped with a positive integral weight $m(e)$ and balanced if for every vertex $v$ of $\mathcal{C}$ we have

$$
\sum_{e: v \prec e} m(e) w_{v, e}=0
$$

The local cone at $v$ is the one-dimensional fan whose rays are spanned by the $w_{w, e}$ and given weight $m(e)$ for $v \prec e$. This is also sometimes referred to as the star of the vertex $v$ (see for example [27]).

Definition 2.4 A tropical curve in $N_{\mathbb{R}}$ is a one dimensional $\Lambda$-rational polyhedral complex equipped with weights on its edges that satisfies the balancing condition, up to the equivalence relation generated by subdivision of edges preserving the weights.

A tropical curve $X$ in a tropical toric variety $N_{\Delta}$ is the closure in $N_{\Delta}$ of a tropical curve $X^{\circ}$ in $N_{\mathbb{R}}$.

$X \backslash X^{\circ}$ is a finite set, whose points we consider as vertices of $X$ and call the infinite vertices. The edges of $X$ are the closures of the edges of $X^{\circ}$.

A $\Lambda$-metric graph (which we will often just call a metric graph) is, roughly speaking, a finite graph in which every edge $e$ has a positive length $l_{e} \in \Lambda \cup\{\infty\}$. We allow loop edges, meaning edges whose endpoints agree and half open edges, i.e. edges which have only one endpoint. If $l_{e} \in \Lambda_{>0}$, we view $e$ as an interval of length $e$. Half open edges are identified with $\mathbb{R}_{\geq 0}$. Leaf edges are the only edges that are allowed to have infinite length and are identified with $[0, \infty]$ with the topology of a closed interval. For a more precise account on metric graphs, we refer the reader to $[1$, Section 2.1]. 

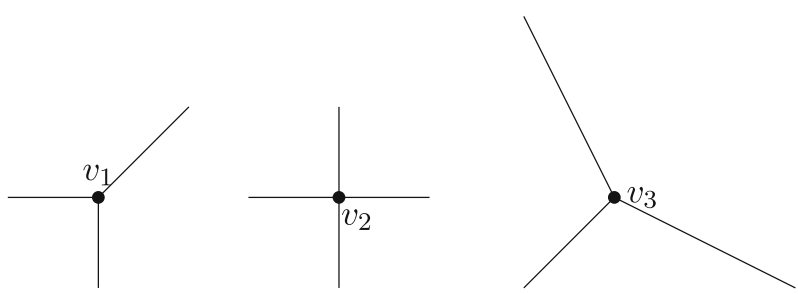

Fig. 1 The types of vertices in tropical curves in $\mathbb{R}^{2}$. The vertex on the left is smooth, the other two vertices are not smooth

By an edge of a metric graph $\Gamma$ we mean an edge in some graph model $G$ of $\Gamma$. For

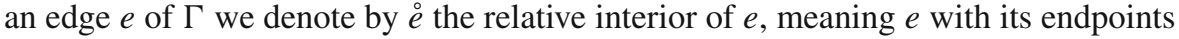
removed. For two points $x, y \in \stackrel{\odot}{e}$ we denote by $d_{e}(x, y)$ their distance in $\stackrel{\circ}{e}$. (Note that this might not be the distance in $\Gamma$, as there might be a shorter path that leaves $\stackrel{\circ}{\text {. }) ~}$

We call a metric graph finite if all its edges have finite length.

Example 2.5 A tropical curve in $N_{\mathbb{R}}$ has a canonical structure as a metric graph where the length of an edge is given by the lattice length, meaning the length of the primitive vector $w_{v, e}$ equals 1 .

A tropical curve $X$ in a tropical toric variety $N_{\Delta}$ is not necessarily a metric graph since two infinite rays might meet at infinity, creating a vertex at infinity which does not have valence 1 . However, $X$ is a metric graph if every point in $X \backslash X^{\circ}$ has exactly one adjacent edge.

Definition 2.6 An edge in a tropical curve is smooth if its weight is 1 . A finite vertex $v$ is smooth if $\left\langle w_{v, e} \mid v \prec e\right\rangle_{\mathbb{Z}}$ is a saturated lattice of $\operatorname{rank} \operatorname{val}(v)-1$ in $N$, where $\operatorname{val}(v)$ is the number of edges adjacent to $v$. An infinite vertex is smooth if it has one adjacent edge. A vertex that is not smooth is called singular. A tropical curve is smooth if all its edges and vertices are smooth.

Remark 2.7 Following [17] a tropical variety is smooth if it is locally isomorphic to the Bergman fan of a matroid.

A one-dimensional weighted fan in $\mathbb{R}^{n}$ is the Bergman fan of a matroid if and only if it is isomorphic to the fan whose rays are spanned by $x_{1}, \ldots, x_{n}$ and $-\sum_{i=1}^{n} x_{i}$ and all weights are 1. Thus Definition 2.6 agrees with the one in [17] for the case of curves.

Example 2.8 Consider the tropical curves in Fig. 1. Each of them depicts a vertex in a tropical curve in $\mathbb{R}^{2}$ with lattice $N=\mathbb{Z}^{2}$. In the leftmost picture, the outgoing directions are $(-1,0),(0,-1)$ and $(1,1)$, which span $\mathbb{Z}^{2}$, thus $v_{1}$ is a smooth vertex. In the picture in the middle, the span of the primitive vectors is again $\mathbb{Z}^{2}$, but there are 4 vertices adjacent to $v_{2}$, thus $v_{2}$ is not smooth. In the picture on the right, the outgoing directions are $(2,-1),(-1,2)$ and $(-1,-1)$. The span of these vectors is $\left\{(x, y) \in \mathbb{Z}^{2} \mid x-y\right.$ is divisible by 3$\}$. This has rank 2 , but is not saturated in $\mathbb{Z}^{2}$, thus $v_{3}$ is not a smooth vertex. 


\subsection{Berkovich curves and their extended skeleta}

Let $X$ be a variety over $K$. The associated Berkovich space [3] is $X^{\text {an }}:=\left\{x=\left.\left(p_{x},|\cdot|_{x}\right)\left|p_{x} \in X,\right| \cdot\right|_{x}\right.$ is an absolute value on $k\left(p_{x}\right)$ extending $\left.|\cdot|_{K}\right\}$

with the topology such that the canonical forgetful map $X^{\text {an }} \rightarrow X$ is continuous and for all open subsets $U$ of $X$ and $f \in \mathcal{O}(U)^{\times}$the map $U^{\text {an }} \rightarrow \mathbb{R},\left(p_{x},\left.||\right|_{x}.\right) \mapsto\left|f\left(p_{x}\right)\right|_{x}$ is continuous. We will often write $|f(x)|:=\left|f\left(p_{x}\right)\right|_{x}$. If $X=\operatorname{Spec}(A)$ is an affine variety then

$$
X^{\text {an }}=\left\{|.| \text { multiplicative seminorm on } A \text { extending }|\cdot|_{K}\right\}
$$

with the topology such that for all $f \in A$ the map $X^{\text {an }} \rightarrow \mathbb{R} ;|.| \mapsto|f|$ is continuous. For morphism $\varphi: X \rightarrow Y$ of $K$-varieties we obtain a morphism $\varphi^{\text {an }}: X^{\text {an }} \rightarrow Y^{\text {an }}$.

Now let $X$ be a curve over $K$. For $x \in X^{\text {an }}$ we denote by $\mathscr{H}(x)$ the completion of $k\left(p_{x}\right)$ with respect to $|.|_{x}$ and by $\widetilde{\mathscr{H}(x)}$ its residue field. Following Berkovich and Thuillier [3,32] we say $x$ is of type I if $p_{x} \in X(K)$ and of type II if $p_{x}$ is the generic point of $X$ and $\operatorname{trdeg}[\widetilde{\mathscr{H}(x)}: \tilde{K}]=1$. If $x$ is of type I, then $|\cdot|_{x}=|\cdot|_{K}$, thus the forgetful map $X^{\text {an }} \rightarrow X$ induces a bijection from the set of type I points of $X^{\text {an }}$ onto $X(K)$. We will thus identify $X(K)$ with the subset of $X^{\text {an }}$ that consists of type I points. If $x$ is of type II, then we denote by $C_{x}$ the smooth projective $\tilde{K}$-curve with function field $\widetilde{\mathscr{H}(x)}$ and by $g(x)$ its genus, which we call the genus of $x$.

We now recall the notion of completed skeleta of $X^{\text {an }}$, which is due to Baker, Payne and Rabinoff [5].

Definition 2.9 We consider $\mathbb{A}^{1}=\operatorname{Spec} K[T]$. For $-\infty \leq s<r \in \mathbb{R}$ denote

$$
B(r)=\left\{\left.x \in \mathbb{A}^{1, \text { an }}|\log | T\right|_{x}<r\right\} \text { and } A(r, s)=\left\{\left.x \in \mathbb{A}^{1, \text { an }}|s<\log | T\right|_{x}<r\right\} .
$$

We call $B(r)$ an open disc of logarithmic radius $r$ and $A(r, s)$ a generalized open annulus of logarithmic radii $s$ and $r$. We call $A(r, s)$ an annulus with logarithmic radii $s$ and $r$ if $s \in \mathbb{R}$ and a punctured disc of radius $r$ if $s=-\infty$. We call $r-s \in \mathbb{R} \cup \infty$ the length of $A(r, s)$.

We denote by $\rho_{B(t)}$ the element of $B(t)$ defined by $\left|\sum a_{i} T^{i}\right|_{\rho_{B(t)}}=\max _{i}\left|a_{i}\right| t^{i}$ and call the set

$$
\Sigma(A(r, s))=\left\{\rho_{B(t)} \mid s<\log t<r\right\}
$$

the skeleton of $A(r, s)$. There is a canonical retraction $\tau: A(r, s) \rightarrow \Sigma(A(r, s))$ which is a strong deformation retraction.

Definition 2.10 Let $X$ be a smooth projective curve over $K$. A completed semistable vertex set $V$ of $X$ is a finite subset of $X^{\text {an }}$ consisting of type I and II points such that $X^{\text {an }} \backslash V$ is isomorphic to a disjoint union of finitely many generalized open annuli and infinitely many open discs. 
For a completed semistable vertex set $V$ of $X^{\text {an }}$ there is a canonical associated subspace $\Sigma(V)$ of $X^{\text {an }}$, called the completed skeleton $\Sigma(V)$, which is a metric graph. There is a canonical retraction $\tau_{V}: X^{\text {an }} \rightarrow \Sigma(V)$, such that $\Sigma(V)$ is a strong deformation retract of $X^{\text {an }}$. As the name suggests, the vertex set of $\Sigma_{V}$ is $V$. The edges are the skeleta of the generalized open annuli that are connected components of $X^{\text {an }} \backslash V$. The length of such an edge is the length of the corresponding annulus.

If $X$ is projective and $V$ is a completed semistable vertex set that only consists of type II points, we call $V$ a semistable vertex set and $\Sigma(V)$ a finite skeleton of $X$. A finite skeleton is a finite metric graph and we will often denote it by $\Gamma$.

Let $V$ be a completed semistable vertex set of $X$. Then the set of type II points in $V$ forms a semistable vertex set for $X$. We call the associated finite skeleton the finite part of $\Sigma(V)$ and denote it by $\Sigma(V)_{\text {fin }}$.

Definition 2.11 A smooth projective curve of genus $g>0$ is called Mumford curve if for some semistable vertex set $V$ the skeleton $\Gamma(V)$ has first Betti number equal to $g$.

Remark 2.12 Note that since $\Gamma(V)$ is a deformation retract of $X^{\text {an }}$, the first Betti number of $\Gamma(V)$ is independent of $V$. Thus we might replace "some" by "every" in Definition 2.11. Furthermore $X$ is a Mumford curve if and only if $g(x)=0$ for all type II points $x$ in $X^{\text {an }}$. Another equivalent definition of Mumford curve is that any point $x \in X^{\text {an }}$ has a neighborhood that is isomorphic to an open subset of $\mathbb{P}^{1 \text {, an }}[20$, Proposition $2.26 \&$ Theorem 2.28].

\subsection{Tropicalization of curves}

Let $Y$ be a toric variety with dense torus $T$. Let $N$ be the cocharacter lattice of $T$, $N_{\mathbb{R}}:=N \otimes \mathbb{R}$ and $\Delta$ the fan in $N_{\mathbb{R}}$ associated to $Y$.

Definition 2.13 The tropicalization of $Y$ is

$$
\operatorname{Trop}(Y):=N_{\Delta}
$$

There is a canonical tropicalization map trop: $Y^{\text {an }} \rightarrow \operatorname{Trop}(Y)$, which is a continuous proper map of topological spaces [30, Section 3].

We assume that the reader is familiar with tropicalizations of closed subvarieties of algebraic tori $[15,28]$. Here we consider tropicalizations of closed subvarieties of toric varieties, which may be seen as a compactification of the latter. We quickly sketch the relation: Given a closed embedding $\varphi: X \rightarrow Y$ of a smooth projective curve $X$ into a toric variety $Y$ that meets the dense torus $T$, denote by $X^{\circ}:=\varphi^{-1}(T)$. Then $\operatorname{Trop}_{\varphi}\left(X^{\circ}\right)$ is a dense open subset of $\operatorname{Trop}_{\varphi}(X)$ and we obtain the latter from the former by putting points at the end of the unbounded edges.

Example 2.14 If $Y=\mathbb{G}_{m}^{n}$ is a torus of dimension $n$ with fixed coordinates, then $\Delta$ is only the origin in $\mathbb{R}^{n}$ and we have $\operatorname{Trop}(Y)=\mathbb{R}^{n}$. The restriction of the map trop: $\mathbb{G}_{m}^{n, \text { an }} \rightarrow \mathbb{R}^{n}$ to $\mathbb{G}_{m}^{n}(K)=\left(K^{*}\right)^{n}$ is the usual tropicalization map $X(K) \rightarrow$ $\mathbb{R}^{n} ; x \mapsto\left(\log \left|x_{1}\right|_{K}, \ldots, \log \left|x_{n}\right|_{K}\right)$. 
If $Y=\mathbb{P}^{1}$, then Example 2.2 shows that $\operatorname{Trop}\left(\mathbb{P}^{1}\right)$ is homeomorphic to a closed interval. Since it contains a one-dimensional vector space as a dense open subset, a good point of view is $\operatorname{Trop}\left(\mathbb{P}^{1}\right)=[-\infty, \infty]$ with the topology of a closed interval.

The map trop: $\mathbb{P}^{1, \text { an }} \rightarrow \operatorname{Trop}\left(\mathbb{P}^{1}\right)$ is then given by $\left(p,|\cdot|_{x}\right) \mapsto \log |z(p)|_{x}$, where $z$ is the coordinate function on $\mathbb{P}^{1}$.

Remark 2.15 For two toric varieties $Y_{1}$ and $Y_{2}$, we have $\operatorname{Trop}\left(Y_{1} \times Y_{2}\right)=\operatorname{Trop}\left(Y_{1}\right) \times$ $\operatorname{Trop}\left(Y_{2}\right)$. This holds because the fan of $Y_{1} \times Y_{2}$ is the product of the fans of $Y_{1}$ and $Y_{2}$.

Let $X$ be a curve over $K$. For a closed embedding $\varphi: X \rightarrow Y$ we denote $\varphi_{\text {trop }}:=$ trop $\circ \varphi^{\text {an }}$ and $\operatorname{Trop}_{\varphi}(X):=\varphi_{\text {trop }}\left(X^{\text {an }}\right)$ the associated tropicalization of $X$. One can define canonical weights on $\operatorname{Trop}_{\varphi}(X)$ that make it into a tropical curve in $\operatorname{Trop}(Y)$ in the sense of Definition 2.4 (see for example [15]). We will define these weights in Definition 2.18.

Definition 2.16 If $Y^{\prime}$ is another toric variety, $\varphi^{\prime}: X \rightarrow Y^{\prime}$ is another closed embedding and $\pi: Y^{\prime} \rightarrow Y$ is a morphism of toric varieties, there exists a canonical map $\operatorname{Trop}\left(Y^{\prime}\right) \rightarrow \operatorname{Trop}(Y)$, which is linear on the dense subset $N_{\mathbb{R}}$ and maps $\operatorname{Trop}_{\varphi^{\prime}}(X)$ onto $\operatorname{Trop}_{\varphi}(X)$. We call $\varphi^{\prime}$ a refinement of $\varphi$.

Note that refinements yield the inverse system in Payne's result that the inverse limit of all tropicalizations is homeomorphic to $X^{\text {an }}$ [30, Theorem 4.2].

\subsection{Factorization skeleta}

Let $\varphi: X \rightarrow Y$ be a closed embedding of a smooth projective curve $X$ into a toric variety $Y$ that meets the dense torus $T$. Denote by $X^{\circ}:=\varphi^{-1}(T)$ the preimage of the dense torus.

Definition 2.17 Let $\Sigma(\varphi)$ be the set of points in $X^{\text {an }}$ that do not have an open neighborhood that is isomorphic to an open disc and contained in $\left(X^{\circ}\right)^{\text {an }}$. We call $\Sigma(\varphi)$ the completed skeleton associated to $\varphi$.

The set $\Sigma(\varphi)$ is indeed a completed skeleton for $X$ [5, Theorem 4.22]. We denote by $\tau_{\varphi}: X^{\text {an }} \rightarrow \Sigma(\varphi)$ the retraction.

Baker, Payne and Rabinoff show that we have a commutative diagram

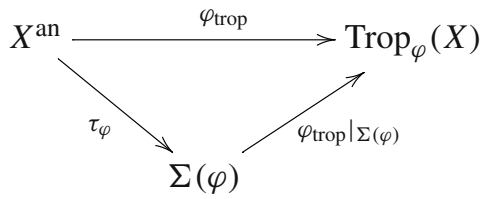

and that $\left.\varphi_{\text {trop }}\right|_{\Sigma(\varphi)}$ is linear on each edge of $\Sigma(\varphi)[6$, Lemma $5.3 \&$ Proposition 5.4 (1)].

We can subdivide $\operatorname{Trop}_{\varphi}(X)$ and $\Sigma(\varphi)$ in such a way that each edge of $\Sigma(\varphi)$ is either contracted to a point or mapped homeomorphically to an edge of $\operatorname{Trop}_{\varphi}(X)[6$, 
Lemma 5.4. (2)]. Let $e$ be an edge in $\operatorname{Trop}_{\varphi}(X)$. Let $e_{1}, \ldots, e_{k}$ be the edges of $\Sigma(\varphi)$ mapping homeomorphically to $e$. For each $i$, we fix $x_{i} \neq y_{i} \in \stackrel{\circ}{e}_{i}$.

Definition 2.18 We call

$$
m\left(e_{i}\right)=\frac{d_{e}\left(\varphi_{\text {trop }}\left(x_{i}\right), \varphi_{\text {trop }}\left(y_{i}\right)\right)}{d_{e_{i}}\left(x_{i}, y_{i}\right)} \text { and } m(e)=\sum_{i=1}^{k} m\left(e_{i}\right)
$$

the stretching factor of $\left.\varphi_{\text {trop }}\right|_{e_{i}}$ and the weight of $e$, respectively.

The definition of weight agrees with the usual one (see for example [15, Definition 3.14]) by [6, Corollary 5.9].

Proposition 2.19 Let $\varphi: X \rightarrow Y$ be a closed embedding of $X$ into a toric variety that meets the dense torus $T$ and $\Sigma(\varphi)$ the associated skeleton. Denote by $X^{\circ}:=\varphi^{-1}(T)$. Then the following are equivalent:

(i) $\varphi_{\text {trop }}$ maps $\Sigma(\varphi)$ homeomorphically onto its image and is an isometry when restricted to $\Sigma(\varphi) \cap X^{\circ}$, an .

(ii) The map $\left.\varphi_{\text {trop }}\right|_{\Sigma(\varphi)}: \Sigma(\varphi) \rightarrow \operatorname{Trop}_{\varphi}(X)$ is injective and all weights on $\operatorname{Trop}_{\varphi}(X)$ are 1 .

Proof Assume that (ii) holds. The map $\left.\varphi_{\text {trop }}\right|_{\Sigma(\varphi)}$ is surjective, thus bijective. Since it is a bijective map between compact Hausdorff spaces, it is a homeomorphism. Hence both (i) and (ii) imply that $\left.\varphi_{\operatorname{trop}}\right|_{\Sigma(\varphi)}$ is a homeomorphism onto its image.

Thus it remains to show that if $\left.\varphi_{\text {trop }}\right|_{\Sigma(\varphi)}$ is a homeomorphism it is an isometry when restricted to $\Sigma(\varphi) \cap X^{\circ}$, an if and only if all weights on $\operatorname{Trop}_{\varphi}(X)$ are all equal to one. This follows from Definition 2.18.

Definition 2.20 We say that $\varphi_{\text {trop }}$ is fully faithful if the equivalent conditions of Proposition 2.19 hold.

The notion of fully faithful tropicalization is stronger then the notion of faithful tropicalization introduced by Baker, Payne and Rabinoff [6]. It is also slightly stronger then the notion of totally faithful tropicalization introduced by Cheung, Fantini, Park and Ulirsch [9] (see also [8]). The difference is that a totally faithful tropicalization only needs to be an isometry when restricted to $\Sigma(\varphi) \cap X^{\circ \text {, an }}$. Note however that the authors of [9] mainly work in the situation of tropical compactifications and in this case the notions of totally faithful and fully faithful agree.

\subsection{Rational functions and divisors on metric graphs}

Let $\Gamma$ be a finite $\Lambda$-metric graph. A point $x \in \Gamma$ is called $\Lambda$-rational if its distance from some, or equivalently every, vertex is in $\Lambda$. A rational function on $\Gamma$ is a piecewise linear function $F: \Gamma \rightarrow \mathbb{R}$ with integer slopes all of whose points of non-linearity are $\Lambda$-rational. A divisor on $\Gamma$ is a finite formal linear combination of $\Lambda$-rational points. 
Its degree is the sum of the coefficients. We denote by $\operatorname{Div}(\Gamma)$ the group of divisors. For a rational function $F$ its divisor is

$$
\operatorname{div}(F):=\sum \lambda_{i} x_{i} \text { where } \lambda_{i}:=\sum_{e: x_{i} \prec e} d_{e} F\left(x_{i}\right)
$$

and $d_{e} F\left(x_{i}\right)$ is the outgoing slope of $F$ along the edge $e$ at $x_{i}$. We call $\operatorname{div}(F)$ a principal divisor on $\Gamma$. We denote by $\operatorname{Prin}(\Gamma)$ the group of principal divisors on $\Gamma$.

Let $X$ be a smooth projective curve and $\Gamma$ a finite skeleton with retraction $\tau$. Let $f$ be in $K(X)^{*}$. Then $F:=\left.\log |f(x)|\right|_{\Gamma}$ is a rational function on $\Gamma$ and $\tau_{*}(\operatorname{div}(f))=\operatorname{div}(F)$ [5, Theorem 5.15] (see also [32, Proposition 3.3.15] for the same result phrased in a slightly different language).

Definition 2.21 We say that edges $e_{1}, \ldots, e_{g}$ form the complement of a spanning tree of $\Gamma$ if there exists a graph model $G$ for $\Gamma$ with set of edges $E$ such that $e_{i} \in E$ and the subgraph of $G$ spanned by the edges $E \backslash\left\{e_{1}, \ldots, e_{g}\right\}$ is connected, contractible and contains all vertices of $G$.

Note that in this definition, $g$ is necessarily the first Betti number of $\Gamma$.

The notion of break divisor was introduced by Mikhalkin, and Zharkov [29]. They observed that any degree $g$ divisor on a metric graph has a unique break divisor in its rational equivalence class (see Theorem 2.23). Break divisors were also studied in detail by An, Baker, Kuperberg, and Shokrieh, who also study discrete versions [2].

Definition 2.22 Let $\Gamma$ be a metric graph and $g=\operatorname{dim}_{\mathbb{R}} \mathrm{H}^{1}(\Gamma, \mathbb{R})$ its first Betti number. A break divisor is a degree $g$ effective divisor $B=p_{1}+\cdots+p_{g}$ such that there exist edges $e_{1}, \ldots, e_{g}$ that form the complement of a spanning tree of $\Gamma$ such that $p_{i} \in e_{i}$.

Theorem 2.23 (Mikhalkin - Zharkov) Let $D$ be a degree $g$ divisor on $\Gamma$. Then there exists a unique break divisor $B$ on $\Gamma$ such that $D-B \in \operatorname{Prin}(\Gamma)$.

Break divisors will play an important role in Theorem 3.2, which we will use to prove our main theorems, as well as to construct tropicalizations in honeycomb form for elliptic curves (see Example 3.5). In our applications we will deal with break divisors that are supported on two-valent points of $\Gamma$. If $B$ is such a break divisor then $\Gamma \backslash \operatorname{supp}(B)$ is connected and contractible.

We will see in Example 3.6 that it is really necessary to restrict to break divisors that are supported on two-valent points in Theorem 3.2.

\section{Lifting theorem}

In this section $X$ is a smooth projective Mumford curve of genus $g$ over $K$. We fix a semistable vertex set $V$ with corresponding finite skeleton $\Gamma$ and retraction $\tau$. We denote by $J_{0}(X):=\left\{[D] \in \operatorname{Pic}(X) \mid \tau_{*} D \in \operatorname{Prin}(\Gamma)\right\}$.

Proposition 3.1 Let $B=p_{1}+\cdots+p_{g}$ be a break divisor on $\Gamma$ that is supported on two-valent points and write $R_{i}=\tau^{-1}\left(p_{i}\right) \cap X(K)$. Then for all $\mathcal{Y}=\left(y_{1}, \ldots, y_{g}\right) \in$ 
Fig. 2 An edge $e$ with four pillar points $p_{1}, p_{2}, p_{3}$ and $p_{4}$ and a piecewise linear function with divisor $p_{1}-p_{2}-p_{3}+p_{4}$

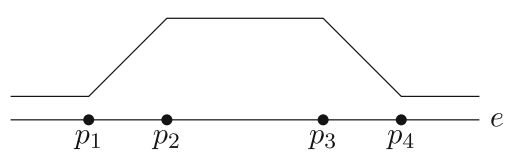

$R_{1} \times \cdots \times R_{g}$ the map

$$
\begin{aligned}
\varphi \mathcal{Y}: R_{1} \times \cdots \times R_{g} & \rightarrow J_{0}(X) \\
\left(x_{1}, \ldots, x_{g}\right) & \mapsto \sum_{i=1}^{g}\left[x_{i}-y_{i}\right]
\end{aligned}
$$

is a surjection.

Proof We consider [7, Proof of Theorem 1.1]. Baker and Rabinoff work in the same setup, but for them $X$ is any curve, not necessarily a Mumford curve. Thus in their situation both the set of $\mathcal{Y}$ they allow and the domain of $\varphi \mathcal{Y}$ is $\left(R_{1} \times \cdots \times R_{b}\right) \times C^{*}$. Here $b$ is the first Betti number of the skeleton of $X$ and $C^{*}=\prod_{x \in X^{\text {an }} ; g(x)>0} C_{x}(\tilde{K})^{g(x)}$. An element $\mathcal{Y} \in\left(R_{1} \times \cdots \times R_{b}\right) \times C^{*}$ is denoted by $\left(\mathcal{Y}_{1}, \mathcal{Y}_{2}\right)$ for $\mathcal{Y}_{1} \in R_{1} \times \cdots \times R_{b}$ and $\mathcal{Y}_{2} \in C^{*}$. They show that $\varphi_{\left(\mathcal{Y}_{1}, \mathcal{Y}_{2}\right)}$ is surjective when $\mathcal{Y}_{2}$ is generic. If $X$ is a Mumford curve, then $b=g$ and $C^{*}$ is just a one point set. Thus $\mathcal{Y}_{2}$ is automatically generic and our proposition follows.

Theorem 3.2 Let $D \in \operatorname{Div}(X)$ of degree $g$ and $B=p_{1}+\cdots+p_{g} \in \operatorname{Div}(\Gamma)$ a break divisor such that $\tau_{*} D-B$ is a principal divisor on $\Gamma$. Assume that $B$ is supported on two-valent points of $\Gamma$. Then there exist $x_{i} \in X(K)$ such that $\tau_{*} x_{i}=p_{i}$ and such that $D-\sum_{i=1}^{g} x_{i}$ is a principal divisor on $X$.

Proof Let $y_{i} \in X(K)$ such that $\tau_{*} y_{i}=p_{i}$. We have $\left[D-\sum_{i=1}^{g} y_{i}\right] \in J_{0}(X)$. Thus by Proposition 3.1 there exist $x_{i} \in \tau^{-1}\left(p_{i}\right) \cap X(K)$ such that $\left[D-\sum_{i=1}^{g} y_{i}\right]=$ $\left[\sum_{i=1}^{g}\left(x_{i}-y_{i}\right)\right]$. In other words $\left[D-\sum_{i=1}^{g} x_{i}\right]=0$ which means that $D-\sum_{i=1}^{g} x_{i}$ is a principal divisor on $X$.

Definition 3.3 Let $e$ be an edge of $\Gamma$. Four points $p_{1}, p_{2}, p_{3}, p_{4} \in \stackrel{\circ}{e}$ are called pillar points in $e$ if they are $\Lambda$-rational, $d_{e}\left(p_{1}, p_{2}\right)=d_{e}\left(p_{3}, p_{4}\right)$ and for $i=2,3$ we have $\left[p_{i-1}, p_{i}\right] \cap\left[p_{i}, p_{i+1}\right]=p_{i}$ (See Fig. 2.)

Figure 2 shows the graph of a piecewise linear function whose divisor is $p_{1}-p_{2}-$ $p_{3}+p_{4}$. In particular that divisor is principal.

Corollary 3.4 Let $D \in \operatorname{Div}^{0}(X)$ such that $\tau_{*} D$ is a principal divisor on $\Gamma$. Let $e_{1}, \ldots, e_{g}$ be edges that form the complement of a spanning tree of $\Gamma$. Fixing pillar points $p_{i, 1}, p_{i, 2}, p_{i, 3}, p_{i, 4}$ in $\stackrel{\circ}{i}_{i}$ there exist $x_{i j} \in X(K)$ such that $\tau\left(x_{i j}\right)=p_{i j}$ and $f \in K(X)^{*}$ such that $\operatorname{div}(f)=D+\sum_{i=1}^{g}\left(x_{i, 1}+x_{i, 4}\right)-\sum_{i=1}^{g}\left(x_{i, 2}+x_{i, 3}\right)$.

Proof The divisor $\sum_{i=1}^{g}\left(p_{i, 1}+p_{i, 4}\right)-\sum_{i=1}^{g}\left(p_{i, 2}+p_{i, 3}\right)$ is principal on $\Gamma$, thus so is $\tau_{*} D+\sum_{i=1}^{g}\left(p_{i, 1}+p_{i, 4}\right)-\sum_{i=1}^{g}\left(p_{i, 2}+p_{i, 3}\right)$. Thus, for $j=1,3$, 4, fixing $x_{i j}$ such that $\tau_{*} x_{i j}=p_{i j}$ and writing $D^{\prime}=D+\sum_{i=1}^{g}\left(x_{i, 1}+x_{i, 4}\right)-\sum_{i=1}^{g} x_{i, 3}$ and 
$B=p_{1,2}+\cdots+p_{g, 2}$, we find that $\tau_{*} D^{\prime}-B$ is a principal divisor on $\Gamma$. Since $B$ is a break divisor supported on two-valent points, applying Theorem 3.2 to $D^{\prime}$ and $B$ we get the result.

Example 3.5 (Tate curves) Chan and Sturmfels use theta functions to produce nice tropicalizations of elliptic curves [11] (see also [6, Theorem 6.2]). In this example we show how Theorem 3.2 can be used to construct such nice tropicalizations combinatorially.

Let $E$ be an elliptic curve with bad reduction. We will use Theorem 3.2 to construct a closed embedding $\varphi: E \rightarrow \mathbb{P}^{2}$ whose tropicalization looks like the right hand side of Fig. 3, which Chan and Sturmfels call symmetric honeycomb form.

The minimal skeleton $\Gamma_{\min }$ is a circle. We pick three points $q_{1}, q_{2}, q_{3} \in \Gamma_{\min }$ that are equidistant from each other. Our skeleton $\Gamma$ is obtained from $\Gamma_{\min }$ by adding edges of length $d\left(q_{i}, q_{j}\right) / 2$ at each of the $q_{i}$, denoting their endpoints by $p_{i}$. We subdivide each edge $\left[q_{i}, q_{j}\right]$ at its midpoint and label our new vertices as on the left hand side of Fig. 3. The solid part of the figure is now our skeleton $\Gamma$.

We pick points $x_{1,1} \neq x_{1,2}, x_{2,1} \neq x_{2,2}, x_{3,1} \neq x_{3,2}$ and $x_{6} \in E(K)$ such that $\tau\left(x_{i, j}\right)=p_{i}$ and $\tau\left(x_{6}\right)=p_{6}$.

Let $D_{1}=-x_{1,1}+x_{2,1}-x_{2,2}+x_{3,1}-x_{6}$. Then $\tau_{*} D_{1}=-p_{1}+p_{3}-p_{6}$ and $\tau_{*} D_{1}+p_{4}=\operatorname{div}\left(F_{1}\right)$ for a rational function $F_{1}$ on $\Gamma$.

Now applying Theorem 3.2 to $-D_{1}$ and $p_{4}$ we obtain a function $f_{1} \in K(E)^{*}$ and $x_{4} \in E(K)$ such that $\tau\left(x_{4}\right)=p_{4}$ and $\operatorname{div}\left(f_{1}\right)=D_{1}+x_{4}$. We normalize $f_{1}$ such that $F_{1}=\left.\log \left|f_{1}\right|\right|_{\Gamma}$.

Similarly let $D_{2}=-x_{1,1}+x_{1,2}-x_{2,2}+x_{3,2}-x_{6}$ then $\tau_{*} D_{2}=-p_{2}+p_{3}-p_{6}$ and $\tau_{*} D_{2}+p_{5}=\operatorname{div}\left(F_{2}\right)$, for a rational function $F_{2}$ on $\Gamma$. We obtain a function $f_{2} \in K(E)^{*}$ and $x_{5} \in E(K)$ such that $\tau\left(x_{5}\right)=p_{5}$ and $\operatorname{div}\left(f_{2}\right)=D_{2}+x_{5}$.

Let $\varphi$ be the morphism associated to the rational map $\left[f_{1}: f_{2}: 1\right]: E \rightarrow \mathbb{P}^{2}$. By construction, the graph on the left hand side of Fig. 3, including the dashed lines, which are infinite edges, is the associated completed skeleton $\Sigma(\varphi)$. We write $G_{i}=$ $\log \left|f_{i}\right|_{\Sigma(\varphi)}$. Note that $\left.G_{i}\right|_{\Gamma}=F_{i}$. Further, $\left.\varphi_{\text {trop }}\right|_{\Sigma(\varphi)}=\left(G_{1}, G_{2}\right)$. Thus $\operatorname{Trop}_{\varphi}(E)=$ $\left(G_{1}, G_{2}\right)(\Sigma(\varphi))$ is the tropical curve on the right hand side of Fig. 3.

The functions $f_{1}, f_{2}, 1$ are linearly independent over $K$, since $f_{1}$ is not constant on the zeros of $f_{2}$. Thus by the Riemann-Roch theorem, they form a basis of $L(D)$ where $D=x_{1,1}+x_{2,2}+x_{6}$. Since $D$ is very ample by [16, Corollary IV.3.2(b)], this shows that $\varphi$ is a closed embedding.

Example 3.6 In the same example, we can also see that Theorem 3.2 does not hold if we do not require $B$ to be supported on two-valent points. Let $D=p_{1}$. Then the unique break divisor that is linearly equivalent to $D$ is $B=q_{1}$. However we cannot find $x$ and $y$ such that $\tau(x)=p_{1}$ and $\tau(y)=q_{1}$ such that $x-y$ is principal, since no difference of two distinct points is principal on an elliptic curve. 

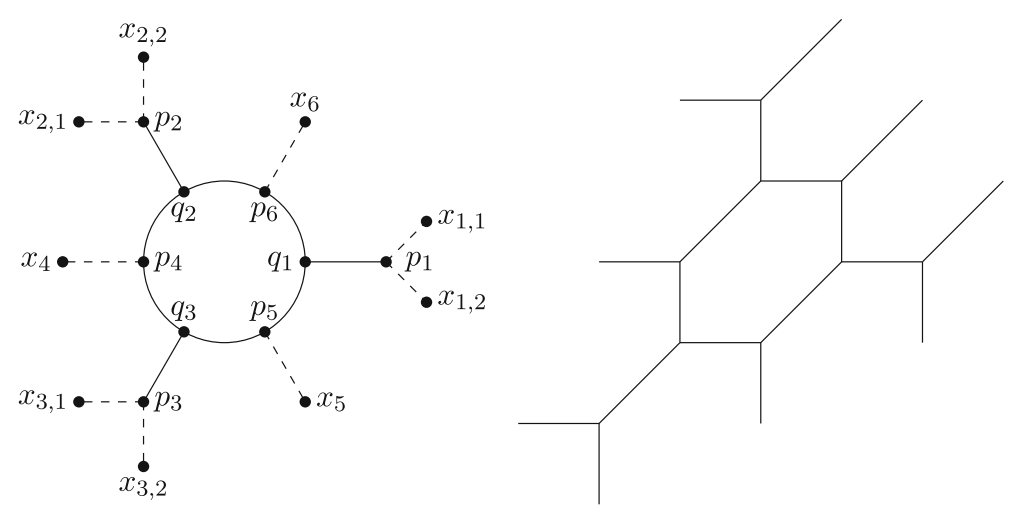

Fig. 3 The skeleton and tropicalization of a Tate curve

\section{Fully faithful and smooth tropicalizations}

\subsection{Describing tropicalizations using extended skeleta}

Let $X$ be smooth projective curve of genus $g>0$. Let $V$ be a minimal semistable vertex set of $X$ with associated finite skeleton $\Gamma$ and retraction $\tau$.

Definition 4.1 Let $\Sigma$ be a completed skeleton of $X$ with retraction $\tau_{\Sigma}, f \in K(X)^{*}$ and write $\operatorname{div}(f)=\sum \pm x_{i}$. Then $f$ is said to be faithful with respect to $\Sigma$ if we have $\tau_{\Sigma}\left(x_{i}\right) \neq \tau_{\Sigma}\left(x_{j}\right)$ for all $i \neq j$.

Note that this implies that $f$ has only simple poles and zeros.

Construction 4.2 Let $\varphi: X \rightarrow Y$ be a closed embedding of $X$ into a toric variety $Y$ that meets the dense torus. Let $\Sigma(\varphi)$ be the completed skeleton associated to $\varphi$. Let $f \in K(X)^{*}$ be faithful with respect to $\Sigma(\varphi)$. Consider the induced closed embedding $\varphi^{\prime}=(\varphi, f): X \rightarrow Y \times \mathbb{P}^{1}$.

We obtain the associated skeleton $\Sigma\left(\varphi^{\prime}\right)$ for $\varphi^{\prime}$ by adding infinite rays $\left[x_{i}, \tau_{\varphi}\left(x_{i}\right)\right]$ for all $x_{i} \in \operatorname{supp}(\operatorname{div}(f))$. We denote by $\tau_{\varphi^{\prime}}$ the associated retraction.

We have the following diagram

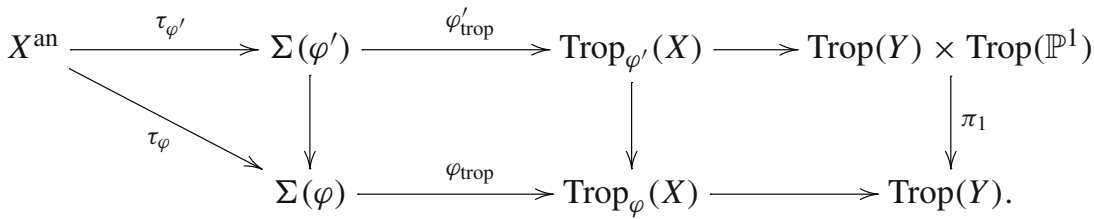

The map on the left contracts the edges $\left[x_{i}, \tau_{\varphi}\left(x_{i}\right)\right]$ to $\tau_{\varphi}\left(x_{i}\right)$. The map $\pi_{1}$ on the right is forgetting the last coordinate.

Thus we obtain $\operatorname{Trop}_{\varphi^{\prime}}(X)$ from $\operatorname{Trop}_{\varphi}(X)$ in two steps:

(i) Take the graph of $\log |f|$ restricted to $\Sigma(\varphi)$. 


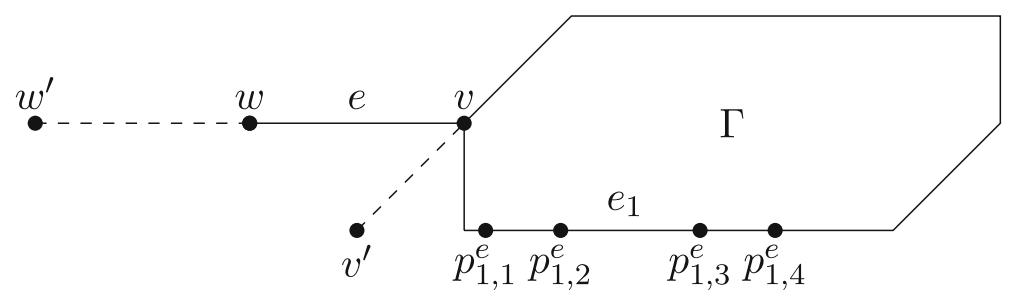

Fig. 4 Situation in Construction 4.4. The dashed lines are infinite edges and solid lines are finite edges

(ii) Add the images of the edges $e_{i}=\left[x_{i}, \tau\left(x_{i}\right)\right]$. These are infinite rays from $\left(\varphi_{\text {trop }}\left(x_{i}\right), \log \left|f\left(x_{i}\right)\right|\right)$ to $\left(\varphi_{\text {trop }}\left(x_{i}\right), \pm \infty\right)$ where the sign of $\infty$ is the opposite of the sign of $x_{i}$ in $\operatorname{div}(f)$.

Lemma 4.3 In the situation of Construction 4.2 , every edge e in $\Sigma\left(\varphi^{\prime}\right)$ that is not an edge of $\Sigma(\varphi)$ is infinite and satisfies $m(e)=1$.

Proof The edge $e$ has to be infinite since we only added infinite rays to $\Sigma(\varphi)$ in Construction 4.2. Since $f$ has only simple poles and zeros, the slope of $\log |f|$ along $e$ is equal to one, thus the corresponding expansion factor equals one.

\subsection{Fully faithful tropicalization}

Throughout this section, $X$ is a Mumford curve and $\varphi: X \rightarrow Y$ a closed embedding of $X$ into a toric variety that meets the dense torus. In this section, we prove Theorem $\mathrm{B}$ from the introduction, showing that $\varphi$ has a refinement that is fully faithful.

We fix a minimal semistable vertex set $V$ and denote by $\Gamma$ the corresponding finite skeleton of $X$ with retraction $\tau$. For our completed skeleton $\Sigma(\varphi)$ associated to $\varphi$ we denote the retraction by $\tau_{\varphi}$ and the finite part by $\Sigma(\varphi)_{\text {fin }}$.

We will now construct for an edge $e$ a function $f_{e} \in K(X)^{*}$ such that the slope of $\log \left|f_{e}\right|$ is equal to 1 along $e$ and such that $f_{e}$ is faithful with respect to $\Sigma(\varphi)$.

Construction 4.4 Let $e$ be a finite edge of $\Sigma(\varphi)$ that is not in $\Gamma$. We label the endpoints $v$ and $w$ in such a way that $w$ and $\Gamma$ lie in different connected components of $\Sigma(\varphi) \backslash v$ (see Fig. 4). Let $v^{\prime}, w^{\prime} \in X(K)$ be such that $\tau_{\varphi}\left(v^{\prime}\right)=v$ and $\tau_{\varphi}\left(w^{\prime}\right)=w$. We fix edges $e_{1}, \ldots, e_{g}$ that form the complement of a spanning tree of $\Sigma(\varphi)$ and pillar points $p_{i j}^{e}$ in $e_{i}$. Applying Corollary 3.4 to $\Sigma(\varphi)_{\text {fin }}$ and $D^{\prime}=v^{\prime}-w^{\prime}$ we obtain $f_{e} \in K(X)^{*}$ such that $\operatorname{div}\left(f_{e}\right)=v^{\prime}-w^{\prime}+\sum \pm x_{i j}^{e}$. By construction $f_{e}$ is faithful with respect to $\Sigma(\varphi)$ and the slope of $\log \left|f_{e}\right|$ along $e$ is 1 . Replacing $f_{e}$ by $a^{-1} \cdot f_{e}$ where $a \in K$ such that $\left|f_{e}(v)\right|=|a|$ we may assume $\log \left|f_{e}(v)\right|=0$.

Construction 4.5 Let $e$ be an infinite edge of $\Sigma(\varphi)$ with finite vertex $v$ and infinite vertex $w^{\prime}$. Let $v^{\prime}$ be a point in $X(K)$ such that $\tau_{\varphi}\left(v^{\prime}\right)=v$ (see Fig. 5). We fix edges $e_{1}, \ldots, e_{g}$ that form the complement of a spanning tree of $\Sigma(\varphi)_{\text {fin }}$ and pillar points $p_{i j}^{e}$ in $e_{i}$. Applying Corollary 3.4 to $\Sigma(\varphi)_{\text {fin }}$ and $D=v^{\prime}-w^{\prime}$ we obtain $f_{e} \in K(X)^{*}$ that is faithful with respect to $\Sigma(\varphi)$ and such that $\log \left|f_{e}\right|$ has slope 1 along $e$. We again normalize such that $\log \left|f_{e}(v)\right|=0$. 


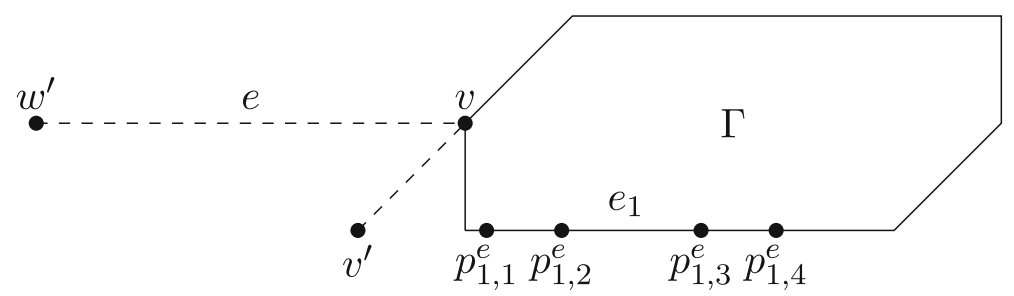

Fig. 5 Situation in Construction 4.5. The dashed lines are infinite edges and solid lines are finite edges

Theorem 4.6 Let $X$ be a Mumford curve. Let $\varphi: X \rightarrow Y$ be a closed embedding of $X$ into a toric variety that meets the dense torus. Then there exists a refinement $\varphi^{\prime}: X \rightarrow Y^{\prime}$ for $\varphi$ that is fully faithful.

Proof Recall that we fixed a finite skeleton of $\Gamma$ of $X$. By [6, Theorem 1.1] we may assume, after possibly replacing $\varphi$ by a refinement, that the map $\left.\varphi_{\text {trop }}\right|_{\Gamma}$ is an isometry onto its image.

Let $E$ be the set of edges of $\Sigma(\varphi)$ that are not in $\Gamma$. The strategy of proof will be as follows: For each edge $e \in E$, we apply Construction 4.4 (if $e$ is a finite edge) or Construction 4.5 (if $e$ is an infinite edge). We make sure that the pillar points we choose to apply these constructions do not get in the way of each other (condition (iii) below) and do not interfere with $e$ after tropicalization (condition (ii) below). This yield a rational function $f_{e}$ for each $e \in E$. We then check that the corresponding embedding $\left(\varphi,\left(f_{e}\right)_{e \in E}\right): X \rightarrow Y \times\left(\mathbb{P}^{1}\right)^{|E|}$ is fully faithful.

For each $i=1, \ldots, g, j=1, \ldots, 4$ and $e \in E$ we pick $p_{i j}^{e} \in \Gamma$ such that

(i) for all $e \in E$ there are edges $e_{i}^{e}, i=1, \ldots, g$, that form the complement of a spanning tree of $\Sigma(\varphi)$ and $p_{i, 1}^{e}, \ldots, p_{i, 4}^{e}$ are pillar points in $e_{i}^{e}$;

(ii) $\varphi_{\text {trop }}\left(\left[p_{i, 1}^{e}, p_{i, 4}^{e}\right]\right) \cap \varphi_{\text {trop }}(e)=\emptyset$ for all $i=1, \ldots, g$;

(iii) $\left[p_{i, 1}^{e}, p_{i, 4}^{e}\right] \cap\left[p_{i^{\prime}, 1}^{e^{\prime}}, p_{i^{\prime}, 4}^{e^{\prime}}\right]=\emptyset$ for $(e, i) \neq\left(e^{\prime}, i^{\prime}\right)$.

Note that a choice of $p_{i j}^{e}$ that satisfies (ii) is possible since $\varphi_{\text {trop }}(e)$ is a line segment, thus cannot cover a full cycle of $\Gamma$.

Now for all finite (resp. infinite) edges $e \in E$ we apply Construction 4.4 (resp. Construction 4.5) and obtain functions $f_{e} \in K(X)^{*}$.

We consider the closed embedding

$$
\varphi^{\prime}:=\left(\varphi,\left(f_{e}\right)_{e \in E}\right): X \rightarrow Y \times\left(\mathbb{P}^{1}\right)^{|E|} .
$$

Following Construction 4.2, the completed skeleton $\Sigma\left(\varphi^{\prime}\right)$ associated to $\varphi^{\prime}$ is obtained from $\Sigma(\varphi)$ by attaching an infinite edge $e_{i j}^{e}$ at each $p_{i j}^{e}$ and by attaching for each $e \in E$ infinite edges to its finite endpoints. If $e=[v, w]$, we denote these edges by $e_{v}^{e}$ and $e_{w}^{e}$ respectively. We claim that the map

$$
\varphi_{\text {trop }}^{\prime}: \Sigma\left(\varphi^{\prime}\right) \rightarrow \operatorname{Trop}(Y) \times \operatorname{Trop}\left(\mathbb{P}^{1}\right)^{E}
$$


is injective. We denote $F_{e}:=\log \left|f_{e}\right|_{\Sigma\left(\varphi^{\prime}\right)}$. By construction, $\varphi_{\text {trop }}^{\prime}$ is injective when restricted to an edge, since $\left.\varphi_{\text {trop }}\right|_{\Gamma}$ is injective and $F_{e}$ is injective when restricted to $e$ and $e_{i j}^{e}$ for $e \in E$.

To show global injectivity, let us set up some notation. Recall that for each edge

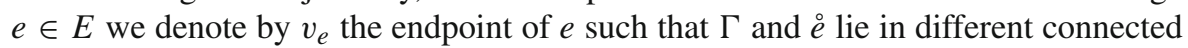
components of $\Gamma \backslash v$ and by $w_{e}$ the other endpoint. Furthermore, $f_{e}$ was normalized in such a way that $F_{e}\left(v_{e}\right)=0$. Recall that $\Gamma$ is a deformation retract of $\Sigma\left(\varphi^{\prime}\right)$. Thus, we may define a partial order on $E$ by declaring $e \leq e^{\prime}$ if " $e$ is closer to $\Gamma$ then $e^{\prime \prime}$, meaning that $\stackrel{\circ}{e}$ and $\stackrel{e}{e}^{\prime}$ lie in the same connected component of $\Sigma\left(\varphi^{\prime}\right) \backslash v_{e}$.

The idea of the proof of injectivity is that for a point $z \in \Sigma\left(\varphi^{\prime}\right) \backslash \Gamma$ we can detect in which edge $e \in E$ it is contained simply by looking at the set of functions $F_{e}$ satisfying $F_{e}(z) \neq 0$. We then do a case by case analysis of the situation.

Now assume $\varphi_{\text {trop }}^{\prime}\left(z_{1}\right)=\varphi_{\text {trop }}^{\prime}\left(z_{2}\right)$ for $z_{1}, z_{2} \in \Sigma\left(\varphi^{\prime}\right)$. This means that $\varphi_{\text {trop }}\left(z_{1}\right)=$ $\varphi_{\text {trop }}\left(z_{2}\right)$ and $F_{e}\left(z_{1}\right)=F_{e}\left(z_{2}\right)$ for all $e \in E$.

Note that we may assume $z_{1} \notin \Gamma$, since if both $z_{1}$ and $z_{2}$ are in $\Gamma$, then we are done since $\varphi_{\text {trop }}$ is already injective on $\Gamma$. Denote

$$
E^{\prime}:=\left\{e \in E \mid F_{e}\left(z_{1}\right) \neq 0\right\}=\left\{e \in E \mid F_{e}\left(z_{2}\right) \neq 0\right\}
$$

Since $F_{e}\left(v_{e}\right)=0$ and $\operatorname{div}\left(F_{e}\right)=v_{e}-w_{e}+\sum_{i=1}^{g}\left(p_{i, 1}^{e}-p_{i, 2}^{e}-p_{i, 3}^{e}+p_{i, 4}^{e}\right)$ we have $F_{e}\left(v_{e}\right)=0, F_{e}\left(w_{e}\right)>0, F_{e}\left(p_{i, 1}^{e}\right)=F_{e}\left(p_{i, 4}^{e}\right)=0$ and $F_{e}$ is constant on the connected components of $\Sigma\left(\varphi^{\prime}\right) \backslash\left(e \cup\left[p_{i, 1}^{e}, p_{i, 4}^{e}\right]\right)$ (see Figure 6). Thus

$$
\operatorname{supp}\left(F_{e}\right)=\bigcup_{e^{\prime} \geq e} e^{\prime} \cup \bigcup_{i=1}^{g}\left[p_{i 1}^{e}, p_{i 4}^{e}\right]
$$

We deduce that $E^{\prime}$ is closed under $\leq$ and non-empty since $z_{1} \notin \Gamma$.

If $\left|E^{\prime}\right|=1$, say $E^{\prime}=\{e\}$, then

$$
z_{1} \in e \cup \bigcup_{i j} e_{i j}^{e} \text { and } z_{2} \in e \cup \bigcup_{i j} e_{i j}^{e} \cup \bigcup_{i}\left[p_{i, 1}^{e}, p_{i, 4}^{e}\right]
$$

In the case $z_{1} \in e$, we have $\varphi_{\text {trop }}\left(z_{2}\right)=\varphi_{\text {trop }}\left(z_{1}\right) \in \varphi_{\text {trop }}(e)$ which forces $z_{2} \in e$ by (ii) above and (4.2). Since $\left.F_{e}\right|_{e}$ is injective, it follows that $z_{1}=z_{2}$.

In the case $z_{1} \in e_{i j}^{e}$, we have $\varphi_{\text {trop }}\left(z_{2}\right)=\varphi_{\text {trop }}\left(z_{1}\right)=p_{i j}^{e}$, thus $z_{2} \in e_{i j}^{e}$ and because $\left.F_{e}\right|_{i j} ^{e}$ is injective we have $z_{1}=z_{2}$.

If $\left|E^{\prime}\right|>1$, then there exists $e \in E$ such that $E^{\prime}=\left\{e^{\prime} \in E \mid e^{\prime} \leq e\right\}$ by (iii) above and (4.1). For the same reason $\left|E^{\prime}\right|>1$ implies $z_{1}, z_{2} \in e$ and consequently $z_{1}=z_{2}$. Thus $\left.\varphi_{\text {trop }}^{\prime}\right|_{\Sigma\left(\varphi^{\prime}\right)}$ is injective.

The stretching factor for all edges of $\Gamma$ is 1 since $\left.\varphi\right|_{e}$ is an isometry onto its image. For all $e \in E$, the stretching factors are equal to 1 since the slope of $f_{e}$ along $e$ is 1 . For all $e_{i j}^{e}$ the stretching factor is equal to 1 by Lemma 4.3. Since $\left.\varphi_{\text {trop }}^{\prime}\right|_{\Sigma\left(\varphi^{\prime}\right)}$ in injective this means all weights are equal to 1 . Thus $\varphi_{\text {trop }}^{\prime}$ represents $\Sigma\left(\varphi^{\prime}\right)$ faithfully. 
Fig. 6 The graph of $\log \left|f_{e}\right|$ on $e_{i}^{e}$ and the adjacent edges $e_{i j}^{e}$ in $\Sigma\left(\varphi^{\prime}\right)$

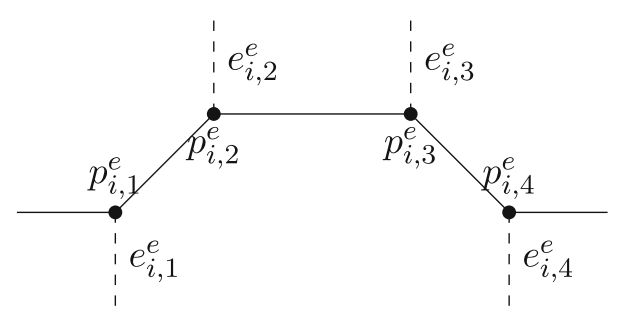

Corollary 4.7 Let $\varphi: X \rightarrow Y$ be a closed embedding of $X$ into a toric variety $Y$ that meets the dense torus. Then there exists a refinement $\varphi^{\prime}$ of $\varphi$ and a section $\psi_{\varphi^{\prime}}: \operatorname{Trop}_{\varphi^{\prime}}(X) \rightarrow X^{\mathrm{an}}$ for $\varphi_{\text {trop }}^{\prime}$.

Proof By Theorem 4.6 we can choose $\varphi^{\prime}$ such that $\varphi_{\text {trop }}^{\prime}$ is fully faithful. Thus $\left.\varphi_{\text {trop }}^{\prime}\right|_{\Sigma\left(\varphi^{\prime}\right)}$ is a homeomorphism and we define $\psi_{\varphi^{\prime}}$ as the composition of the inclusion of $\Sigma\left(\varphi^{\prime}\right)$ into $X^{\text {an }}$ with $\left(\varphi_{\text {trop }}^{\prime} \mid \Sigma\left(\varphi^{\prime}\right)\right)^{-1}$.

\subsection{Smooth tropicalization}

Throughout this section, we will work in the following situation: $X$ is a Mumford curve over $K$ and $\varphi: X \rightarrow Y$ a closed embedding that meets the dense torus such that $\varphi_{\text {trop }}$ is fully faithful. We denote by $\Sigma(\varphi)$ the associated complete skeleton and by $\tau_{\varphi}$ the retraction.

Lemma 4.8 Let $f \in K(X)^{*}$ that is faithful with respect to $\Sigma(\varphi)$. Then $\varphi^{\prime}=$ $(\varphi, f): X \rightarrow Y \times \mathbb{P}^{1}$ is fully faithful. Further, all vertices in $\Sigma\left(\varphi^{\prime}\right)$ that map to singular vertices in $\operatorname{Trop}_{\varphi^{\prime}}(X)$ are contained in $\Sigma(\varphi)$ and map to singular vertices in $\operatorname{Trop}_{\varphi}(X)$.

Proof All edges of $\Sigma\left(\varphi^{\prime}\right)$ that are not edges of $\Sigma(\varphi)$ have expansion factor equal to 1 by Lemma 4.3 . Since $f$ is faithful all these edges have different images under $\tau_{\varphi}$. Since $\varphi_{\text {trop }}$ is fully faithful, they have different images under $\varphi_{\text {trop }}^{\prime}$. Consequently $\left.\varphi_{\text {trop }}^{\prime}\right|_{\Sigma\left(\varphi^{\prime}\right)}$ is injective. Thus $\varphi_{\text {trop }}^{\prime}$ is fully faithful.

Let $v$ be a vertex of $\Sigma\left(\varphi^{\prime}\right)$. Then $v$ is a vertex in $\Sigma(\varphi)$ (after potential subdivision) or infinite. Since $\varphi_{\text {trop }}^{\prime}$ is fully faithfully, the infinite vertices of $\operatorname{Trop}_{\varphi^{\prime}}(X)$ have only one adjacent vertex and are thus smooth. Thus we have to show that if $\varphi_{\text {trop }}(v)$ is a smooth finite vertex of $\operatorname{Trop}_{\varphi}(X)$, then $\varphi_{\text {trop }}^{\prime}(v)$ is a smooth vertex of $\operatorname{Trop}_{\varphi^{\prime}}(X)$.

Let $e_{0}, \ldots, e_{n}$ be the adjacent edges of $v$ and write $w_{i}:=w_{v, e_{i}}$ for the primitive integral vector pointing from $\varphi_{\text {trop }}(v)$ into $\varphi_{\text {trop }}\left(e_{i}\right)$. We denote $F=\left.\log |f|\right|_{\Sigma\left(\varphi^{\prime}\right)}$ and $L(F)=F-F(v)$.

If $v$ is not in $\operatorname{div}(F)$, then $F$ is locally around $\varphi_{\text {trop }}(v)$ the restriction to $\operatorname{Trop}_{\varphi}(X)$ of an affine function on $N_{\mathbb{R}}$. The vertex $v$ still has $n+1$ adjacent edges $e_{0}^{\prime}, \ldots, e_{n}^{\prime}$ in $\Sigma\left(\varphi^{\prime}\right)$ and the primitive vectors are $w_{0}^{\prime}=\left(w_{0}, L(F)\left(e_{0}\right)\right), \ldots, w_{n}^{\prime}=\left(w_{n}, L(F)\left(e_{n}\right)\right)$. Since $F$ has integer slopes and is the restriction of an affine function and the $w_{i}$ span a saturated lattice of rank $n$, so do the $w_{i}^{\prime}$, which shows that $\varphi_{\text {trop }}(v)$ is a smooth vertex of $\operatorname{Trop}_{\varphi^{\prime}}(X)$. 


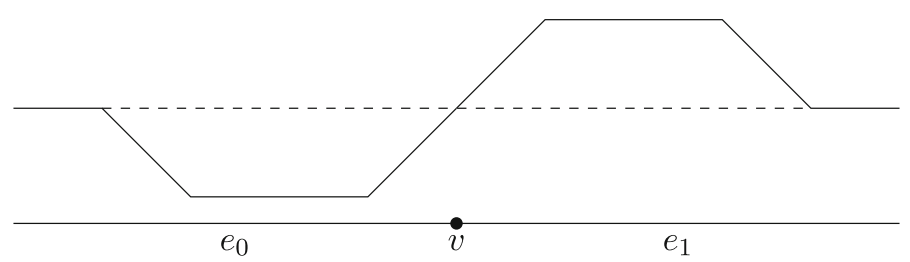

Fig. 7 The graph of $F_{e_{1}}$ along the edges $e_{0}$ and $e_{1}$ in Construction 4.9, with the dashed line being the zero level

If $v \in \operatorname{div}(F)$, since $f$ is faithful with respect to $\Sigma(\varphi), v$ has $n+2$ adjacent edges $e_{0}^{\prime}, \ldots, e_{n}^{\prime}, e_{n+1}^{\prime}$ in $\Sigma\left(\varphi^{\prime}\right)$ and the primitive vectors are

$$
w_{0}^{\prime}=\left(w_{0}, L(F)\left(e_{0}\right)\right), \ldots, w_{n}^{\prime}=\left(w_{n}, L(F)\left(e_{n}\right)\right), w_{n+1}^{\prime}=(0, \pm 1) .
$$

Since the $w_{i}$ span a saturated lattice of rank $n$, the $w_{i}^{\prime}$ span a saturated lattice of rank $n+1$, which shows that $\varphi_{\text {trop }}^{\prime}(v)$ is a smooth vertex of $\operatorname{Trop}_{\varphi^{\prime}}(X)$.

For a vertex $v$ of $\Sigma(\varphi)$ and two adjacent edges $e_{0}$ and $e_{1}$, we now construct a function $f_{e_{1}}$ in $K(X)^{*}$ that we will use to construct a tropicalization that is smooth at $v$. This may be viewed as generalization to any ambient dimension and any vertex of the constructions done for special vertices and ambient dimension 2 by Cueto and Markwig [10, Section 3]

Construction 4.9 Let $v$ be a vertex of $\Sigma(\varphi)$ and let $e_{0}$ and $e_{1}$ be adjacent edges. Let $F_{e_{1}}$ be a piecewise linear function such that $F_{e_{1}}(v)=0, d_{e_{1}} F_{e_{1}}(v)=1, d_{e_{0}} F_{e_{1}}(v)=$ $-1, d_{e} F_{e_{1}}=0$ for all other adjacent edges, $\operatorname{supp}\left(F_{e_{1}}\right) \subset e_{1} \cup e_{0}$, and such that $\operatorname{div}\left(F_{e_{1}}\right)=\sum \pm p_{i}$ for distinct points $p_{i}$ (see Fig. 7). For each $i$ fix $x_{i} \in X(K)$ such that $\tau_{*} x_{i}=p_{i}$ and write $D=\sum \pm x_{i}$. Fixing pillar points $p_{j k}$ outside of $\operatorname{supp}(F)$ and applying Corollary 3.4 we obtain a function $f_{e_{1}} \in K(X)^{*}$ that is faithful with respect to $\Sigma(\varphi)$ and such that the outgoing slope at $v$ of $\log \left|f_{e_{1}}\right|$ equals 1 along $e_{1}$ and -1 along $e_{0}$.

Theorem 4.10 Let $\varphi: X \rightarrow Y$ be an embedding of $X$ into a toric variety $Y$ that meets the dense torus. Then there exists a refinement $\varphi^{\prime}: X \rightarrow Y^{\prime}$ for a toric variety $Y^{\prime}$ such that $\operatorname{Trop}_{\varphi^{\prime}}(X)$ is a smooth tropical curve.

Proof By Theorem 4.6, after replacing $\varphi$ by a refinement, we may assume that $\varphi_{\text {trop }}$ is fully faithful.

Let $v$ be a vertex of $\Sigma(\varphi)$ such that $\varphi_{\text {trop }}(v)$ is a singular vertex of $\operatorname{Trop}_{\varphi}(X)$. Let $e_{0}, \ldots, e_{n}$ be the adjacent edges. For $k=1, \ldots, n$ we pick functions $F_{e_{i}}: \Sigma(\varphi) \rightarrow \mathbb{R}$ as in Construction 4.9. For each $k=1, \ldots, n$ and $i=1, \ldots, g$ we pick pillar points $p_{i, 1}^{k}, p_{i, 2}^{k}, p_{i, 3}^{k}, p_{i, 4}^{k}$ in such a way that

$$
\begin{aligned}
& {\left[p_{i, 1}^{k}, p_{i, 4}^{k}\right] \cap \operatorname{supp}\left(F_{k}\right)=\emptyset \text { for all } i, k \text { and }} \\
& {\left[p_{i, 1}^{k}, p_{i, 4}^{k}\right] \cap\left[p_{i^{\prime}, 1}^{k^{\prime}}, p_{i^{\prime}, 4}^{k^{\prime}}\right]=\emptyset \text { for }(k, i) \neq\left(k^{\prime}, i^{\prime}\right) .}
\end{aligned}
$$


Applying now Construction 4.9, we obtain functions $f_{e_{i}} \in K(X)$. We consider the closed embedding $\varphi^{\prime}:=\left(\varphi,\left(f_{e_{k}}\right)_{k=1, \ldots, n}\right): X \rightarrow\left(\mathbb{P}^{1}\right)^{n}$ and its tropicalization

$$
\varphi_{\text {trop }}^{\prime}: X^{\text {an }} \rightarrow \operatorname{Trop}(Y) \times \operatorname{Trop}\left(\mathbb{P}^{1}\right)^{n}
$$

Applying Lemma $4.3 n$ times, we see that $\varphi_{\text {trop }}^{\prime}$ is fully faithful. By construction $v$ still has $n+1$ adjacent edges $e_{0}^{\prime}, \ldots, e_{n}^{\prime}$ in $\Sigma\left(\varphi^{\prime}\right)$ and $\log \left|f_{e_{i}}\right|$ has slope 1 along $e_{i}^{\prime}$, slope -1 along $e_{0}^{\prime}$, and is constant on the other edges. This means that projecting a neighborhood of $\varphi_{\text {trop }}^{\prime}(v)$ in $\operatorname{Trop}_{\varphi^{\prime}}(X) \subset \operatorname{Trop}(Y) \times \operatorname{Trop}\left(\mathbb{P}^{1}\right)^{n}$ to the second factor, the image is isomorphic to the one-dimensional fan in $\mathbb{R}^{n}$ whose rays are spanned by the coordinate vectors $x_{1}, \ldots, x_{n}$ and their negative sum $x_{0}=-\sum_{i=1}^{n} x_{i}$. Further the primitive vector $w_{v, e_{i}^{\prime}}$ is mapped to $x_{i}$. Thus the $w_{v, e_{i}^{\prime}}$ span a saturated lattice of rank $n$, which means that $v$ is smooth in $\operatorname{Trop}_{\varphi^{\prime}}(X)$.

Since $v$ is singular in $\operatorname{Trop}_{\varphi}(X)$ but not in $\operatorname{Trop}_{\varphi^{\prime}}(X)$, by inductively applying Lemma 4.8, we see that $\operatorname{Trop}_{\varphi^{\prime}}(X)$ has fewer singular points than $\operatorname{Trop}_{\varphi}(X)$.

Thus inductively we can construct $\varphi^{\prime}$ such that $\operatorname{Trop}_{\varphi^{\prime}}(X)$ is smooth.

\section{Only Mumford curves admit smooth tropicalizations}

Let $X$ be a smooth projective curve. In this section we show that the existence of a closed embedding $\varphi: X \rightarrow Y$ such that $\operatorname{Trop}_{\varphi}(X)$ is smooth already implies that $X$ is a Mumford curve. Since we will not change the embedding in this section, we will identify $X$ with its image and simply treat $X$ as a closed subcurve of $Y$. We denote the completed skeleton associated to the inclusion of $X$ into $Y$ by $\Sigma$.

We denote by $K^{\circ}$ the valuation ring of $K$ and by $\tilde{K}$ its residue field. Further we denote by $T$ the dense torus of $Y$, by $N$ its cocharacter lattice and $N_{\Lambda}=N \otimes \Lambda \subset N_{\mathbb{R}}$.

We will use the notion of affinoid domains in $X^{\text {an }}$ and their formal models. For an introduction to these notions we refer the reader to [6, Section 3].

Definition 5.1 Let $w \in N_{\Lambda} \cap \operatorname{Trop}(X)$. Then $X^{w}:=\operatorname{trop}^{-1}(w)$ is an affinoid domain in $X^{\text {an }}$. The point $w$ determines a formal model $\mathcal{X}^{w}$ for $X^{w}$.

The initial degeneration is the special fiber $\operatorname{in}_{w}(X):=\mathcal{X}_{s}^{w}:=\mathcal{X}^{w} \otimes_{K^{\circ}} \tilde{K}$.

Remark 5.2 Assume that $\operatorname{in}_{w}(X)$ is reduced. By [6, Proposition 3.13] we have that $\mathcal{X}^{w}$ is the canonical model of $X^{w}$. Then we have a canonical reduction map red: $X^{w} \rightarrow$ $\operatorname{in}_{w}(X)\left[3\right.$, Section 2.4]. Let $C$ be an irreducible component of $\operatorname{in}_{w}(X)$ with generic point $\eta$. Then there is a unique point $x_{w} \in X^{w}$ such that $\operatorname{red}\left(x_{w}\right)=\eta$ and that point satisfies that $C_{x_{w}}$ is birational to $C$ [3, Proposition 2.4.4]. If $z$ is a smooth closed point of $\operatorname{in}_{w}(X)$ then $\operatorname{red}^{-1}(z)$ is isomorphic to an open disc [4, Proposition 2.2].

In particular, if $\operatorname{in}_{w}(X)$ is smooth and rational, then all type II points in $X^{w}$ have genus 0 .

We will use the following Proposition. Since we will apply it in the case of a trivially valued field, we allow the absolute value of the field to be trivial. 
Proposition 5.3 Let $T$ be an algebraic torus over a non-archimedean field, whose absolute value may be trivial. Let $T^{\prime}$ be a subtorus and let $U$ be a closed subvariety of $T$. If $\operatorname{Trop}(U) \subset \operatorname{Trop}\left(T^{\prime}\right)$ then a translate of $U$ that has the same tropicalization as $U$ is contained in $T^{\prime}$.

Proof We consider the quotient torus $T / T^{\prime}$. Denote by $\bar{U}$ the image of $U$ in the quotient torus $T / T^{\prime}$. Then the tropicalization of $\bar{U}$ in $\operatorname{Trop}\left(T / T^{\prime}\right)=\operatorname{Trop}(T) / \operatorname{Trop}\left(T^{\prime}\right)$ is a point by construction, meaning that $U$ is contained in a translate $t \cdot T^{\prime}$ of $T^{\prime}$, where all entries of $T$ have absolute value 1 . Thus $t^{-1} \cdot U$ is a translate of $U$ that is contained in $T^{\prime}$ and has the same tropicalization as $U$.

In the following, we view $\tilde{K}$ as a non-archimedean field, carrying the trivial absolute value.

Theorem 5.4 Let $T$ be an algebraic torus over $\tilde{K}$. Let $U \subset T$ be a closed curve. If $\operatorname{Trop}(U)$ is smooth then $U$ is smooth and rational.

Proof In the case where $\operatorname{Trop}(U)$ spans $\operatorname{Trop}(T)$, it follows from [23, Proposition 4.2] that the closure of $U$ in $\mathbb{P}^{n}$ is a one-dimensional linear space. Thus $U$ is a smooth rational curve. We reduce to this case: Let $V$ be the vector subspace of $\operatorname{Trop}(T)$ that is spanned by $\operatorname{Trop}(U)$. Since $V$ is a rational subspace, there exists a subtorus $T^{\prime}$ of $T$ such that $\operatorname{Trop}\left(T^{\prime}\right)=V$. Now replacing $U$ by the translate from Proposition 5.3 and applying Katz's and Payne's result to $U$ and $T^{\prime}$ proves the theorem.

Corollary 5.5 If $\operatorname{Trop}(X)$ is smooth, then $\operatorname{in}_{w}(X)$ is a smooth rational curve for all $w \in \operatorname{Trop}(X) \cap N_{\Lambda}$.

Proof Let $w \in \operatorname{Trop}(X) \cap N_{\Lambda}$. Then $\operatorname{in}_{w}(X)$ is a closed subvariety of a torus $T_{\tilde{K}}$ over $\tilde{K}$. Denote by $\operatorname{Trop}\left(\operatorname{in}_{w}(X)\right)$ its tropicalization. Then the local cone at $w$ in $\operatorname{Trop}(X)$ equals $\operatorname{Trop}\left(\operatorname{in}_{w}(X)\right)$ by $[15,10.15]$. Thus $\operatorname{in}_{w}(X)$ is a smooth rational curve by Theorem 5.4 .

Theorem 5.6 If Trop $(X)$ is smooth, then $X$ is a Mumford curve.

Proof Let $w \in \operatorname{Trop}(X) \cap N_{\Lambda}$. By Corollary 5.5, $\operatorname{in}_{w}(X)$ is smooth and rational. Thus all type II points in $X^{w}$ have genus 0 by Remark 5.2. Since all type two points map to $N_{\Lambda}$ under the tropicalization map, all type II points in $X^{\text {an }}$ have genus zero which shows that $X$ is a Mumford curve by Remark 2.12

Theorem 5.7 If $\operatorname{Trop}(X)$ is smooth, then the tropicalization map is fully faithful.

Proof By Corollary 5.5, all initial degenerations are smooth and rational. For all $w \in$ $N_{\Lambda} \cap \operatorname{Trop}(X)$, by Remark 5.2, there is a unique point $x_{w} \in X^{w}$ that satisfies that $\operatorname{red}\left(x_{w}\right)$ is the generic point of $\operatorname{in}_{w}(X)$. Furthermore, every point in $X^{w} \backslash\left\{x_{w}\right\}$ has a neighborhood isomorphic to an open disc, thus is not contained in $\Sigma$. We conclude that every point $w \in N_{\Lambda} \cap \operatorname{Trop}(X)$ has $x_{w}$ as its unique preimage under trop $\left.\right|_{\Sigma}$. Since trop $\left.\right|_{\Sigma}$ is continuous and linear on each edge, this implies that trop $\left.\right|_{\Sigma}: \Sigma \rightarrow \operatorname{Trop}(X)$ is bijective. Since all weights are 1, this shows that the tropicalization map is fully faithful. 
Note that when $X$ comes by base change from a family of Riemann surfaces over the punctured disc, Theorems 5.6 and 5.7 are consequences of [17, Corollary 2]. The relation between Hodge and Betti numbers in tropical geometry is different than in complex geometry. The $(0,1)$-tropical Hodge number of $\operatorname{Trop}(X)$ is equal to the first Betti number of Trop $(X)$. Using this and [17, Corollary 2] one finds that the first Betti number of $\operatorname{Trop}(X)$ is equal to $g$, which, $\operatorname{since} \operatorname{Trop}(X)$ is smooth, implies that trop $\left.\right|_{\Sigma}$ is injective, hence bijective.

Acknowledgements Open Access funding provided by Projekt DEAL. The author was inspired to reconsider the questions in this paper by a question asked by Hannah Markwig during an open problem session at the program "Tropical geometry, amoebas and polytopes" at the Institute Mittag-Leffler. He would like to thank Hannah Markwig for the encouragement and the Institute Mittag-Leffler for the wonderful working conditions. He would also like to thank Matt Baker, Walter Gubler, Yoav Len, Hannah Markwig, Sam Payne, Joe Rabinoff, Veronika Wanner and Annette Werner for helpful discussions and comments. He would also like to thank the anonymous referees for their precise reports and detailed comments.

Open Access This article is licensed under a Creative Commons Attribution 4.0 International License, which permits use, sharing, adaptation, distribution and reproduction in any medium or format, as long as you give appropriate credit to the original author(s) and the source, provide a link to the Creative Commons licence, and indicate if changes were made. The images or other third party material in this article are included in the article's Creative Commons licence, unless indicated otherwise in a credit line to the material. If material is not included in the article's Creative Commons licence and your intended use is not permitted by statutory regulation or exceeds the permitted use, you will need to obtain permission directly from the copyright holder. To view a copy of this licence, visit http://creativecommons.org/licenses/by/4.0/.

\section{References}

1. Amini, O., Baker, M., Brugallé, E., Rabinoff, J.: Lifting harmonic morphisms I: metrized complexes and Berkovich skeleta. Res. Math. Sci., 2:Art. 7, 67 (2015)

2. An, Y., Baker, M., Kuperberg, G., Shokrieh, F.: Canonical representatives for divisor classes on tropical curves and the matrix-tree theorem. Forum Math. Sigma, 2:e24, 25 (2014)

3. Berkovich, V.G.: Spectral theory and analytic geometry over non-Archimedean fields, volume 33 of Mathematical Surveys and Monographs. American Mathematical Society, Providence, RI (1990)

4. Bosch, S., Lütkebohmert, W.: Stable reduction and uniformization of abelian varieties. I. Math. Ann. 270(3), 349-379 (1985)

5. Baker, M., Payne, S., Rabinoff, J.: On the structure of non-Archimedean analytic curves. In Tropical and non-Archimedean geometry, volume 605 of Contemp. Math., pages 93-121. Amer. Math. Soc., Providence, RI (2013)

6. Baker, M., Payne, S., Rabinoff, J.: Nonarchimedean geometry, tropicalization, and metrics on curves. Algebr. Geom. 3(1), 63-105 (2016)

7. Baker, M., Rabinoff, J.: The skeleton of the Jacobian, the Jacobian of the skeleton, and lifting meromorphic functions from tropical to algebraic curves. Int. Math. Res. Not. IMRN 16, 7436-7472 (2015)

8. Cartwright, D., Dudzik, A., Manjunath, M., Yao, Y.: Embeddings and immersions of tropical curves. Collect. Math. 67(1), 1-19 (2016)

9. Cheung, M.-W., Fantini, L., Park, J., Ulirsch, M.: Faithful realizability of tropical curves. Int. Math. Res. Not. IMRN 15, 4706-4727 (2016)

10. Cueto, M.A., Markwig, H.: How to repair tropicalizations of plane curves using modifications. Exp. Math. 25(2), 130-164 (2016)

11. Chan, M., Sturmfels, B.: Elliptic curves in honeycomb form. In Algebraic and combinatorial aspects of tropical geometry, volume 589 of Contemp. Math., pages 87-107. Amer. Math. Soc., Providence, RI, (2013)

12. Gunn, Trevor, Jell, Philipp: Construction of fully faithful tropicalizations for curves in ambient dimension 3. (2019). arxiv:1912.02648

13. Gubler, W., Rabinoff, J., Werner, A.: Skeletons and tropicalizations. Adv. Math. 294, 150-215 (2016) 
14. Gubler, W., Rabinoff, J., Werner, A.: Tropical skeletons. Ann. Inst. Fourier (Grenoble) 67(5), 19051961 (2017)

15. Gubler, W.: A guide to tropicalizations. In Algebraic and combinatorial aspects of tropical geometry, volume 589 of Contemp. Math., pages 125-189. Amer. Math. Soc., Providence, RI, (2013)

16. Hartshorne, R.: Algebraic geometry. Springer-Verlag, New York-Heidelberg, 1977. Graduate Texts in Mathematics, No. 52 (1977)

17. Itenberg, I., Katzarkov, L., Mikhalkin, G., Zharkov, I.: Tropical homology. Math. Ann. 374(1-2), 963-1006 (2019)

18. Jell, P., Rau, J., Shaw, K.: Lefschetz $(1,1)$-theorem in tropical geometry. Épijournal Geom. Algébrique, 2:Art. 11 (2018)

19. Jell, P., Shaw, K., Smacka, J.: Superforms, tropical cohomology, and Poincaré duality. Adv. Geom. 19(1), 101-130 (2019)

20. Jell, P., Wanner, V.: Poincaré duality for the tropical Dolbeault cohomology of non-archimedean Mumford curves. J. Number Theory 187, 344-371 (2018)

21. Katz, E., Markwig, H., Markwig, T.: The $j$-invariant of a plane tropical cubic. J. Algebra 320(10), 3832-3848 (2008)

22. Katz, E., Markwig, H., Markwig, T.: The tropical $j$-invariant. LMS J. Comput. Math. 12, 275-294 (2009)

23. Katz, E., Payne, S.: Realization spaces for tropical fans. In Combinatorial aspects of commutative algebra and algebraic geometry, volume 6 of Abel Symp., pages 73-88. Springer, Berlin (2011)

24. Kawaguchi, S., Yamaki, K.: Effective faithful tropicalizations associated to linear systems on curves. 2016. arxiv:1612.01098, to appear in Memoirs of the American Mathematical Society (2016)

25. Manjunath, M.: Tropical graph curves. (2016). arxiv:1603.08870.pdf

26. Mikhalkin, G.: What are tropical counterparts of algebraic varieties? Oberwolfach Reports 5(2), 1460$1462(2008)$

27. Maclagan, D., Sturmfels, B.: Introduction to tropical geometry. Book in progress, draft available at http://homepages.warwick.ac.uk/staff/D.Maclagan/papers/papers.html

28. Maclagan, D., Sturmfels, B.: Introduction to tropical geometry, volume 161 of Graduate Studies in Mathematics. American Mathematical Society, Providence, RI (2015)

29. Mikhalkin, G., Zharkov, I.: Tropical curves, their Jacobians and theta functions. In Curves and abelian varieties, volume 465 of Contemp. Math., pages 203-230. Amer. Math. Soc., Providence, RI (2008)

30. Payne, S.: Analytification is the limit of all tropicalizations. Math. Res. Lett. 16(3), 543-556 (2009)

31. Shaw, K.: A tropical intersection product in matroidal fans. SIAM J. Discrete Math. 27(1), 459-491 (2013)

32. Thuillier, A.: Théorie du potentiel sur les courbes en géométrie analytique non archimédienne. Applications à la théorie d'Arakelov. (2005). https://tel.archives-ouvertes.fr/file/index/docid/48750/filename/ tel-00010990.pdf

33. Wagner, T.: Faithful tropicalization of Mumford curves of genus two. Beitr. Algebra Geom. 58(1), 47-67 (2017)

Publisher's Note Springer Nature remains neutral with regard to jurisdictional claims in published maps and institutional affiliations. 